\title{
Idosos, infecções sexualmente transmissíveis e aids: conhecimentos e percepção de risco
}

\section{Elderly, sexually transmitted infections and aids: knowledge and risk perception}

\author{
Nívea Maria Izidro de Brito ${ }^{1}$, Smalyanna Sgren da Costa Andrade ${ }^{1}$, Fernanda Maria Chianca da Silva ${ }^{1}$, \\ Marta Regina Chaves Camilo Fernandes ${ }^{1}$, Karen Krystine Gonçalves Brito', Simone Helena dos Santos Oliveira \\ 'Universidade Federal da Paraíba (UFPB) - João Pessoa (PB), Brasil.
}

DOI: http://dx.doi.org/10.7322/abcshs.v41i3.902

\begin{abstract}
RESUMO
Introdução: No contexto das doenças crônicas transmissíveis, a pandemia da aids vem gerando discussões em âmbito internacional sobre as formas de controlar seu avanço, inclusive na população idosa, a qual apresenta incidência considerável de casos. Objetivo: Investigar o conhecimento e verificar a percepção de risco de idosos quanto à contaminação por Infecções Sexualmente Transmissíveis (IST) e HIV. Métodos: Tratase de estudo descritivo de natureza quantitativa realizado com 55 idosos participantes de grupos em duas unidades de saúde da família interligadas à rede-escola. Resultados: A maioria dos idosos era entre 60-70 anos, sexo masculino, casados, católicos, com o nível fundamental incompleto. Além disso, $40 \%$ dos idosos citaram o uso do preservativo como principal método de prevenção às infecções sexuais, 21,9\% responderam que o HIV é transmitido de uma pessoa para outra por meio do contato sexual e $38,2 \%$ citaram que a doença não tem cura. Sobre a percepção de risco, 76,4\% referiram que não tinham nenhuma possibilidade de adquirir Infecções Sexualmente Transmissíveis ou HIV. Tal fator pode contribuir para que essa população se considere pouco vulnerável à contaminação ou não se perceba em risco, o que os torna susceptíveis ao perigo da infecção, favorecendo o aumento do índice de idosos infectados no cenário nacional. Conclusão: Cabe aos organismos governamentais e não governamentais investir em práticas educativas, onde idosos possam ser inseridos em um ambiente que aborde a sexualidade, proporcionando maior segurança e qualidade de vida aos nossos cidadãos.
\end{abstract}

Palavras-chave: idoso; doenças sexualmente transmissíveis; HIV.

\begin{abstract}
Introduction: In the context of chronic diseases, aids pandemic has generated discussions at the international level on methods to control its progress, including in the elderly population, which presents significant incidence of cases. Objective: To investigate the knowledge and verify the perception of risk among the elderly individuals concerning contamination by Sexually Transmitted Infections (STI) and HIV. Methods: This is a quantitative descriptive study, which was conducted with 55 elderly who are participants of groups in two family health units that are interconnected to a school network. Results: Most of the elderly was aged between 60-70 years, male, married, catholic, and with incomplete elementary educational level. Additionally, $40 \%$ of the elderly reported the use of condoms as a primary method of prevention of sexually transmitted infections, $21.9 \%$ of them answered that HIV is sexually transmitted from one person to another by means of sexual contact, and $38.2 \%$ mentioned that disease has no cure. On the perception of risk, $76.4 \%$ reported that they had no possibility of acquiring sexually transmitted infections or HIV. This factor may contribute to a misperception of this population of being less vulnerable and to the unawareness about the risks, making them susceptible to the infection, which may also increase the rate of elderly individuals infected on the national scene. Conclusion: Government and nongovernmental organizations should invest in educational practices that enable the insertion of the elderly in a context to address sexuality, providing protection and quality of life for our citizens.
\end{abstract}

Keywords: aged; sexually transmitted diseases; HIV.

Recebido em: 17/09/2015

Revisado em: 06/04/2016

Aprovado em: 06/05/2016

Autor para correspondência: Smalyanna Sgren da Costa Andrade - Programa de Pós-Graduação em Enfermagem - Centro de Ciências da Saúde Universidade Federal da Paraíba - Cidade Universitária, Campus V - CEP: 58051-900 - João Pessoa (PB), Brasil. E-mail: nana_sgren@hotmail.com Conflito de interesses: nada a declarar. 


\section{INTRODUÇÃO}

Recentemente, a World Health Organization (WHO) apontou que o programa brasileiro de combate à aids (Acquired Immune Deficiency Syndrome) é reconhecido internacionalmente. Suas ações são exitosas do ponto de vista da redução da transmissibilidade da doença. Isso ocorre devido à adoção de medidas inovadoras de prevenção e da cobertura gratuita no tratamento, garantido pelo Sistema Único de Saúde (SUS) ${ }^{1}$.

O órgão mundial ainda cita que o Brasil faz uso de novas tecnologias de prevenção e inseriu recentemente em seu programa de combate à doença uma ação de ampliação do tratamento aos adultos com testes positivos de HIV, mesmo sem comprometimento do sistema imunológico. Isso se deve, sobretudo, aos investimentos governamentais em pesquisas científicas e tecnológicas voltadas à epidemia ${ }^{1}$.

No contexto das doenças crônicas transmissíveis, a pandemia da aids vem gerando bastante discussão em âmbito internacional sobre as formas de controlar seu avanço. Desde 1980 até junho de 2013 foram registrados no Brasil, conforme o Sistema de Informações de Agravos de Notificação (Sinan), 686.478 casos dessa doença, sendo 95.516 (13,9\%) ocorrentes na Região Nordeste ${ }^{2}$.

Em relação aos idosos, em 12 anos (1998-2010) houve aumento na incidência da doença de 7,5 em homens e 2,8 em mulheres por 100.000 habitantes no ano de 1998, para uma incidência de 9,4 e 5,1 por 100.000 habitantes em 2010, respectivamente ${ }^{3}$. Apesar do crescimento desenfreado neste perfil de infectados, muito se investe nas estratégias de controle da epidemia para as populações-chaves, como homens que fazem sexo com homens, transexuais, usuários de drogas injetáveis, profissionais do sexo e população presidiária, e a atenção é menos voltada aos outros perfis, como no caso dos idosos. As tímidas ações de combate à doença, especificamente nessa população, confere ao país o risco de ter cada vez mais indivíduos idosos doentes.

Esse panorama reflete o aumento da prática sexual desprotegida entre idosos, demonstrando que o desejo e a sexualidade estão presentes em todas as etapas da vida do ser humano. Essa fase não está isenta de risco, pois não se deve desvincular o idoso das fontes importantes de prazer ${ }^{4}$. Desse modo, políticas destinadas a esse público devem considerar a capacidadefuncional, a necessidade de autonomia, a participação e a autossatisfação, bem como incentivar a prevenção, o cuidado e a atenção à saúde no que tange à sexualidade 5 .

Supõe-se equívoco na assistência em saúde pensar que o avançar da idade e o declínio progressivo da atividade sexual, embora universais para homens e mulheres, são sinônimos. Ao contrário dessa crença, as pessoas em idade avançada são perfeitamente capazes de manter relações sexuais e de sentir prazer, embora existam alterações fisiológicas decorrentes do processo natural do envelhecimento que diminuam essas sensações ${ }^{6}$.

Pensar que o idoso se encontra em uma faixa etária isenta de riscos concorre para a pouca ou quase ausente proposição de estratégias voltadas para favorecer a saúde sexual responsável das mulheres e dos homens idosos ${ }^{7}$. O estigma de que relação sexual é uma atividade própria das pessoas jovens, com boa saúde e fisicamente atraentes, tem demonstrado que a privação sexual vem acompanhada de tabus e preconceitos dos próprios idosos, da família e/ou dos profissionais de saúde, interferindo na vivência sexual saudável.

Quando o idoso vive sua sexualidade sem o uso de preservativos, existe a possibilidade de contaminação por Infecções Sexualmente Transmissíveis (IST). O aumento dos casos das IST na população envelhecida demonstra a fragilidade das campanhas de prevenção direcionadas a essa população no que tange à criação de estratégias que incentivem a utilização de preservativos entre idosos, configurando-se como entrave aos gestores da saúde ${ }^{8}$.

Diante da problemática das IST e da aids para a população idosa e de suas repercussões individuais e coletivas, a relevância deste estudo se encontra na intenção de informar à comunidade científica, à sociedade e aos profissionais de saúde sobre fatores que podem concorrer para contaminação e adoecimento, influenciados pelo conhecimento adquirido ao longo da vida e pela percepção de risco frente à infecção. Assim, explicitar esses fatores como influentes na adoção de medidas preventivas é um passo contribuinte e favorável à redução de novos casos da doença em idosos no país.

O estudo foi norteado pelas seguintes indagações: O que os idosos sabem sobre IST e aids? Quais são suas percepções de risco quanto a essas doenças? Diante dos questionamentos, objetivou-se investigar o conhecimento e verificar a percepção de risco de idosos quanto às IST e HIV.

Cabe mencionar que esta pesquisa é fruto de um plano de trabalho proveniente do projeto de pesquisa intitulado "Educação em saúde e cuidado no contexto das doenças crônicas transmissíveis e não transmissíveis: investigação de estratégias de promoção da saúde", financiado pelo Conselho Nacional de Desenvolvimento Científico e Tecnológico (CNPq). O projeto maior abrange pesquisas desenvolvidas por acadêmicos do curso de graduação em Enfermagem, da Escola Técnica de Saúde e de pós-graduação da Universidade Federal da Paraíba (UFPB).

\section{MÉTODOS}

Trata-se de um estudo descritivo de natureza quantitativa realizado em duas Unidades de Saúde da Família (USF) do município de João Pessoa (PB), que se constituem como cenários de integração com a rede-escola, no âmbito dos serviços de saúde vinculados à Universidade Federal da Paraíba (UFPB).

A amostra por conveniência foi constituída de 55 idosos envolvidos nas atividades das unidades de saúde vinculadas à Escola Técnica de Saúde/UFPB. Essas unidades estão adscritas a duas comunidades carentes da capital paraibana com risco ambiental e condições de insalubridade e violência. Os participantes selecionados faziam parte dos grupos de idosos das USFs vinculados ao HIPERDIA (Programa voltado às pessoas acometidas por hipertensão e/ou diabetes). 
Os critérios de inclusão da amostra foram: idosos que se encontravam orientados e demonstravam facilidade na expressão verbal durante o período de coleta de dados, e aceitação voluntária. Após as atividades dos grupos, os idosos foram convidados a participarem da pesquisa. As entrevistas ocorreram em salas das USFs, para proporcionar maior privacidade e evitar constrangimentos.

O instrumento de coleta de dados, do tipo formulário, conteve questões fechadas, compreendendo a caracterização do perfil sociodemográfico, e abertas, relacionadas ao conhecimento dos idosos e a percepção de risco sobre as IST/HIV/aids.

Em alguns questionamentos a palavra "aids" foi utilizada como sinônimo de HIV para melhorentendimento da pergunta, já que tal termo é mais utilizado no senso comum, apesar de serem terminologias diferentes.

As respostas abertas foram transcritas e resumidas em expressões simples, agrupando e quantificando-as, de forma a constituir categorias de análise. Os dados foram analisados com o auxílio do programa estatístico SPSS (Statistical Package for Social Sciences), versão 20.0. As respostas foram calculadas por meio de frequências absolutas e relativas.

A pesquisa foi aprovada pelo Comitê de Ética em Pesquisa do Centro de Ciências da Saúde (CEP/CCS), conforme protocolo $\mathrm{n}^{\circ}$ 089/11, e CCAE $\mathrm{n}^{\circ}$ 1562.0.000.462-11. Durante a coleta de dados (período de junho a agosto de 2011), foram realizadas orientações aos participantes quanto à finalidade da pesquisa, à garantia de sigilo, ao direito de desistência em qualquer fase da pesquisa e à leitura do Termo de Consentimento Livre e Esclarecido. Na ocasião, atendia-se às exigências da 196/96 (atual Resolução 466/12) do Conselho Nacional de Saúde, que dispõe sobre as normas e diretrizes regulamentadoras de pesquisas envolvendo seres humanos?.

\section{RESULTADOS}

A caracterização sociodemográfica do estudo em tela demonstrou que a maioria encontra-se na faixa etária entre 60-70 anos (60\%), sendo $50,9 \%$ do sexo masculino e $49,1 \%$ do sexo feminino. O estado civil casado predominou em $45,4 \%$, e a religião católica em $54,5 \%$ da amostra do estudo. No que tange à escolaridade, $65,5 \%$ possuem o ensino fundamental incompleto, tendo como atividade do lar 36,4\%.

A Tabela 1 apresenta as respostas dos entrevistados em relação ao questionamento: "Como se previne as Infecções Sexualmente Transmissíveis e o HIV"?

$\mathrm{Na}$ Tabela 2 seguem as respostas referentes à indagação: "Como se transmite Infecções Sexualmente Transmissíveis e HIV?"

$\mathrm{Na}$ Tabela 3 estão as respostas dos idosos referentes à questão: "O que o(a) senhor(a) sabe sobre o tratamento da aids?"

Na Tabela 4 estão as categorias relacionadas à indagação: "Qual o risco de o(a) senhor(a) se contaminar com Infecção Sexualmente Transmissível e HIV?”

\section{DISCUSSÃO}

A percepção que a sociedade tem da pessoa idosa envolve mitos e tabus, o que influencia as práticas de saúde junto à população. Nessa perspectiva ainda há muito que se fazer, haja vista a necessidade de vislumbrar o idoso em todas as suas dimensões, reconhecendo a sexualidade como algo possível de se viver na velhice. Parte desse processo de mudança repousa sobre o próprio conhecimento e consciência do longevo, acerca do HIV/aids.

Tabela 1: Conhecimento dos idosos acerca das medidas preventivas às IST e ao HIV, em João Pessoa, 2012

\begin{tabular}{|l|c|c|}
\hline Categorias & $\mathbf{n}$ & $\%$ \\
\hline Usar preservativo & 22 & 40 \\
\hline Não sabe informar & 11 & 20 \\
\hline Não ter relações sexuais com pessoas infectadas & 4 & 7,2 \\
\hline Conhecer a pessoa com quem possui relação sexual & 2 & 3,6 \\
\hline Outros & 16 & 29,2 \\
\hline Total & 55 & 100 \\
\hline
\end{tabular}

Tabela 2: Conhecimento dos idosos sobre os modos de transmissão das IST e do HIV, em João Pessoa, 2012

\begin{tabular}{|l|c|c|}
\hline Categorias & $\mathbf{n}$ & $\%$ \\
\hline Contato sexual & 12 & 21,9 \\
\hline Contato com o sangue & 11 & 20 \\
\hline Não sabe informar & 8 & 14,5 \\
\hline Outros & 24 & 43,6 \\
\hline Total & 55 & 100 \\
\hline
\end{tabular}

Tabela 3: Conhecimentos dos idosos acerca do tratamento da aids, em João Pessoa, 2012

\begin{tabular}{|l|c|c|}
\hline Categorias & $\mathbf{n}$ & $\%$ \\
\hline Não tem cura & 21 & 38,2 \\
\hline Existe tratamento medicamentoso & 17 & 30,9 \\
\hline Não sabe informar & 17 & 30,9 \\
\hline Total & 55 & 100 \\
\hline
\end{tabular}

Tabela 4: Percepção de risco dos idosos às IST e ao HIV, em João Pessoa, 2012

\begin{tabular}{|l|c|c|}
\hline Categorias & $\mathbf{n}$ & $\%$ \\
\hline Nenhuma & 42 & 76,4 \\
\hline Baixa & 9 & 16,4 \\
\hline Alta & 2 & 3,6 \\
\hline Média & 2 & 3,6 \\
\hline Total & 55 & 100 \\
\hline
\end{tabular}


Partindo da premissa acima elencada, consubstanciam-se os achados desse estudo. Em relação aos modos de prevenção, $40 \%$ dos idosos citaram o uso do preservativo como principal método de prevenção às infecções sexuais e $20 \%$ não souberam informar sobre as medidas preventivas. Releva informar que a categoria Outros agrupou 29,2\% dos respondentes que verbalizaram como medidas preventivas: não sair com prostitutas, não beijar na boca de uma pessoa infectada, não utilizar o mesmo banheiro, evitar contato físico com pessoas que vivem com HIV/aids e evitar o mesmo assento (Tabela 1).

Apesar da maioria dos participantes mencionar a camisinha como o método de prevenção às IST/HIV, muitos idosos não apontaram a utilização do preservativo como forma de prevenção. Esse resultado evidenciou a necessidade de esclarecimentos e orientações aos idosos sobre os meios de prevenção das IST e HIV, a fim de compreenderem as diferentes vias de transmissão e as medidas preventivas a essas enfermidades.

Importa pontuar que a maioria dos participantes possuía o nível fundamental incompleto $(65,5 \%)$. Esse fator pode influenciar no risco de contaminação pelo HIV nessa população, ainda não suficientemente esclarecida quanto ao sexo seguro, visto que a escolarização parece exercer impacto na adoção de práticas saudáveis, dificultando, assim, que muitos idosos recebam orientações necessárias sobre a importância do uso do preservativo.

Um estudo sobre a aids e o grau de escolaridade entre jovens e idosos observou que estes possuíam conhecimento insatisfatório sobre a epidemia. Em outras palavras, os idosos tinham informações insuficientes sobre HIV/aids comparado a adultos jovens em mesmo nível de escolarização ${ }^{10}$.

A resistência ao uso do preservativo por parte dos idosos está associada ao constrangimento em adquiri-lo, ao desconhecimento de como usá-lo, ao medo de perder a ereção efetiva e ao conceito equivocado de que serviria apenas para evitar gravidez ${ }^{11}$. Essa resistência pode favorecer o aumento de novos casos de IST nessa população. Além disso, existe o aumento da utilização do viagra pelos idosos, o que gera novas relações sexuais desprotegidas, ampliando o risco de IST ${ }^{12}$.

Corroborando nossos achados, uma pesquisa sobre o perfil epidemiológico em idosos apontou que os participantes também indicaram o uso da camisinha e não ter relações com pessoas infectadas como medidas de prevenção ${ }^{13}$. O desenvolvimento de programas de saúde pública específicos para a população idosa é uma iniciativa salutar na elucidação das principais dúvidas relacionadas ao HIV/aids, principalmente quando impera a educação em saúde na promoção de mudanças comportamentais ${ }^{14}$.

Estudo semelhante sobre o conhecimento e a percepção do grau de risco quanto às IST/HIV/aids e a utilização do preservativo entre idosos demonstrou que a maioria dos entrevistados não fazia uso do insumo durante as relações sexuais, principalmente as mulheres, devido à dificuldade de negociação com o parceiro ${ }^{15}$.

Portanto, os achados reforçam a necessidade de estratégias educativas que desconstruam crenças errôneas sobre as IST e a aids e que, ao mesmo tempo, reforcem a necessidade da adoção das medidas básicas e comprovadamente mais eficazes na prevenção desses agravos, que é o uso do preservativo.

As características gerais da epidemia da aids têm se manifestado também na população idosa, principalmente por meio do sexo inseguro, em decorrência do aumento da atividade sexual, da disponibilidade de tecnologias que melhorem e prolonguem a performance sexual, bem como a resistência em usar o preservativo ${ }^{13}$. O fato de citarem o preservativo como forma de prevenção às contaminações por infecções sexuais não garante o seu uso entre os idosos.

Essa constatação evoca a reflexão sobre como o compartilhamento de informações relevantes à sexualidade está inserido no processo de trabalho em saúde, ou seja, de forma individualizada ou coletiva, principalmente quando se pensa no aumento da incidência do HIVnessa parcela populacional.

Observou-se que $21,9 \%$ dos participantes responderam que o HIV é transmitido de uma pessoa para outra por meio do contato sexual; $20 \%$ referiram que a transmissão ocorre por meio do contato com o sangue e $14,5 \%$ não sabiam informar sobre as formas de transmissão. A categoria "Outros", com 43,6\%, diz respeito ao agrupamento de concepções errôneas quanto ao modo de transmissão, que são: dividir talheres e louça, utilizar o mesmo banheiro e roupas de cama/banho bem como abraçar e beijar na boca uma pessoa infectada (Tabela 2).

Embora os participantes deste estudo tenham citado as principais formas de transmissão do HIV adequadamente, o entendimento sobre as vias de contaminação é inadequado quando abrange ações cotidianas que não envolvem o contato sanguíneo. Em outras palavras, atividades que fazem parte do imaginário social e que realmente são modos de transmissão em potencial de doenças contagiosas envolvem o contato próximo ou o uso de objetos de uma pessoa infectada por outra saudável. Entretanto, essas formas de transmissão de viroses sazonais coletivas não se aplicam à contaminação pelo HIV, o que pode ter confundido os idosos, devido às crenças apoiadas no senso comum sobre contaminações por doenças virais.

O estudo demonstrou que a carência de informações de um idoso de hoje é reflexo da pouca procura dessas informações pelo adolescente de ontem. Em épocas passadas, os jovens não tinham facilidade de diálogo, tampouco acesso a diversas fontes de informação como nos dias atuais, e privavamse de adequado conhecimento relacionado à sexualidade. A construção de barreira moral entre pais e filhos e a falta de preparo familiar para orientar jovens sobre sexualidade culminaram na carência de informação nos idosos contemporâneos como uma herança intelectual negativa ${ }^{16}$. 
Em consonância com esses achados, uma pesquisa realizada com um grupo de idosos na cidade de Anápolis, no Estado de Goiás, constatou que, apesar do bom nível de conhecimento demonstrado pelos participantes, existiam lacunas sobre as formas de transmissão quanto ao HIV, demonstrando a necessidade de investimentos públicos na educação em saúde para a redução dos riscos ${ }^{17}$.

Outro estudo afirma que, apesar de os participantes possuírem informações importantes sobre a aids, são frequentes as citações sobre formas incorretas de transmissão e modos preventivos influenciados pelo senso comum, cuja crença é de que o contágio pode acontecer facilmente pelo processo de higienização ${ }^{18}$.

Embora 30,9\% dos participantes não soubessem informar se a aids possui ou não tratamento, a maioria dos idosos $(38,2 \%)$ respondeu que a doença não tem cura e 30,9\% referiram que existe tratamento medicamentoso (Tabela 3). Apesar dos esforços do governo federal, dos estados, dos municípios e do Distrito Federal compartilharem elevados investimentos para controlar a multiplicação do vírus na população brasileira (por meio da prevenção, da vigilância e da distribuição de medicamentos a todos aqueles que necessitarem), a população idosa ainda requer ações integradas voltadas para esse segmento, buscando garantir que seus direitos sexuais sejam realizados com segurança.

As campanhas educativas realizadas em massa para tal fim podem alcançar resultados significativos em todos os setores da sociedade, ilustrando a doença de modo a condizer com uma nova realidade. Atualmente, existe tratamento para aids, não com finalidade de cura, mas como forma de controlar a doença e prolongar a vida do indivíduo.

Para o tratamento são usadas drogas chamadas antirretrovirais, que inibem o crescimento e a replicação do vírus, por meio da redução da carga viral na corrente sanguínea. Geralmente são empregados mais de um tipo de antirretroviral para conseguir o efeito desejado ${ }^{19}$. No Brasil, todo cidadão tem direito ao acesso gratuito aos antirretrovirais. A boa adesão ao tratamento é condição indispensável para o controle da doença, com efeitos positivos diretos na melhora da qualidade de vida da pessoa com HIV/aids.

Esses resultados demonstram bom conhecimento em relação ao tratamento de uma doença que atinge milhões de pessoas em todo mundo, confirmando que a Política Brasileira de Enfrentamento da aids, implementada pelo Ministério da Saúde, vem consolidando progressivamente o ideal de descentralização do conhecimento a respeito dessa epidemia.

Sobre a percepção de risco, 76,4\% responderam que não tinham nenhuma possibilidade de adquirir infecções sexualmente transmissíveis ou HIV, seguido por $16,4 \%$ dos idosos que acreditam ter baixo risco de contaminação. Os percentuais para percepção de risco alta e média foram
3,6\% (Tabela 4). As principais justificativas baseavam-se na ausência de ativi dade sexual e na parceria fixa.

Quanto à ausência de atividade sexual, essa razão alegada pelos participantes comunga com o que a vasta literatura acerca da sexualidade em idosos descreve, ou seja, com o passar dos anos, tanto a libido quanto a disposição para manutenção das relações sexuais diminuem devido às condições fisiológicas do processo natural do envelhecimento e às condições clínico-patológicas associadas ao preconceito da vida sexual ativa. Já a parceria fixa torna-se um vilão quando se pensa no risco de contaminação, devido à incerteza da fidelidade do(a) companheiro(a).

Um estudo realizado no sul do país revelou que existem lacunas no conhecimento dos idosos em relação ao conceito de HIV/aids, de formas de transmissão, de vulnerabilidade e de outros fatores que contribuem para a ascensão da doença nessa faixa etária. $\mathrm{Na}$ ocasião, a pesquisa recomendou o desenvolvimento de programas de saúde pública específicos para população em questão, que se dediquem de melhor forma à elucidação das principais dúvidas relacionadas à epidemia ${ }^{20}$.

Práticas de autocuidado e de responsabilização pelo outro podem ser construídas em grupos e nas relações de socialização afetivas e de aprendizagem estabelecidas entre os próprios idosos usuários, as instituições, os serviços, incluindo no cotidiano da assistência em saúde temáticas a respeito da sexualidade e do risco de contaminação às IST e HIV. Isso pode reduzir a conivência de todos com a invisibilidade da percepção de risco e consequente perigo de contaminação pelo vírus ${ }^{21}$.

Conclui-se que hoje o acesso à informação sobre diversos assuntos é facilitado, havendo investimento em veiculações por meio da mídia sobre questões relacionadas à sexualidade e às doenças sexualmente transmissíveis; porém, a população idosa ainda enfrenta grandes barreiras no reconhecimento da sua sexualidade, fator que dificulta a proposição de ações para abordar essa questão, a fim de minimizar os danos à saúde sexual dos idosos.

A análise dos resultados revelou que a maioria dos idosos apresenta "frágeis conhecimentos" sobre os modos de prevenção e de transmissão das doenças sexualmente transmissíveis e da aids, fator que pode contribuir para "não se perceberem em risco ou se perceberem em baixo risco" de contaminação, o que, consequentemente, os tornam suscetíveis ao perigoda infecção, colaborando com o aumento do índice de idosos infectados no cenário nacional.

Portanto, cabe aos organismos governamentais e não governamentais investirem em práticas educativas, onde idosos possam ser inseridos em ambiente que aborde a temática de forma aberta, livre de preconceitos, partindo essencialmente do reconhecimento da sexualidade, proporcionando maior segurança e qualidade de vida aos nossos cidadãos. 


\section{REFERÊNCIAS}

1. World Health Organization (WHO). Consolidated guidelines on HIV prevention, diagnosis, treatment and care for key populations, 2014. Disponível em: http://www.paho.org/bra/images/stories/ Documentos2/eng\%20guias\%20pop\%20vul\%20who-1. pdf?ua=1. Acesso em: 03 mar 2015.

2. Brasil. Ministério da Saúde. Secretaria de Vigilância em Saúde. Departamento de DST, Aids e Hepatites Virais. HIV/AIDS. Bol Epidemiol. 2014;3(1):11-18.

3. Brasil. Ministério da Saúde. Secretaria de Vigilância em Saúde. Departamento de DST, Aids e Hepatites Virais. HIV e AIDS no Brasil. Bol Epidemiol. 2012;1(1):6-13.

4. Vieira DLFC, Sobral B. O corpo envelhece, a sexualidade não: AIDS no diagnóstico diferencial entre as doenças comuns nos idosos. Int Clin Med. 2009;2(B6).

5. VerasR. Envelhecimento populacional contemporâneo: demandas, desafios e inovações. Rev Saúde Pública. 2009;43(3):548-54. http://dx.doi.org/10.1590/S0034-89102009005000025

6. Laroque MF, Affeldt AB, Cardoso DH, Souza GL, Santana MG, Lange C. Sexualidade do Idoso: comportamento para a prevenção de DST/AIDS. Rev Gaúcha Enferm. 2011;32(4):774-80. http://dx.doi.org/10.1590/S1983-14472011000400019

7. Gradim CVC, Sousa AMM, Lobo JM. A prática sexual e o envelhecimento. Cogitare Enferm. 2007;12(2):204-13. http://dx.doi.org/10.5380/ce.v12i2.9826

8. Maschio MBM, Balbino AP, Souza PFR, Kalinke LP. Sexualidade na terceira idade: medidas de prevenção para doenças sexualmente transmissíveis e AIDS. Rev Gaúcha Enferm. 2011;32(3):583-9. http://dx.doi.org/10.1590/S1983-14472011000300021

9. Brasil. Ministério da Saúde. Conselho Nacional de Saúde, Comissão Nacional de Ética em Pesquisa. Resolução $n^{\circ} 466$, 12 de dezembro de 2012: Revoga a Resolução 196/96 sobre as diretrizes e normas regulamentadoras de pesquisa envolvendo seres humanos. Brasília: Ministério da Saúde; 2012.

10. Melo HMA, Leal MCC, Marques APO, Marino JG. O conhecimento sobre Aids de homens idosos e adultos jovens: um estudo sobre a percepção desta doença. Ciênc Saúde Coletiva. 2012;17(1):43-53. http://dx.doi.org/10.1590/S1413-81232012000100007

11. Serra A, Sardinha AHL, Lima SCVS, Pereira ANS. Perfil comportamental de idosos com HIV/AIDS atendidos em um centro de referência. Rev Enferm UFPE. 2013;7(2):407-13. http://dx.doi.org/10.5205/r euol.3049-24704-1-LE.0701201311
12. Sousa JL. Sexualidade na terceira idade: Uma discussão da Aids, envelhecimento e medicamentos para disfunção erétil. DST $J$ Bras Doenças Sex Tansm. 2008;20(1):59-64.

13. Godoy VS, Ferreira MD, Silva EC, Gir E, Canini SRMS. O perfil epidemiológico da Aids em idosos utilizando sistemas de informações em saúde do DATASUS: realidades e desafios. DST J Bras Doenças Sex Tansm. 2008;20(1):7-11.

14. Lazzarotto AR, Kramer AS, Hädrich $M$, Tonin $M$, Caputo $P$, Sprinz, E. O conhecimento de HIV/aids na terceira idade: estudo epidemiológico no Vale do Sinos, Rio Grande do Sul, Brasil. Ciêno Saúde Coletiva. 2008; 3(6):1833-40.

http://dx.doi.org/10.1590/S1413-81232008000600018

15. Garcez BS, Garcez JS, Paixão MRP, Fernandes ACV, Santos RB. Conhecimento e percepção do grau de risco sobre DST e HIV/ AIDS e a utilização do preservativo entre idosos: o autocuidado sadio e a enfermagem neste contexto. Rev Pesq Cuid Fundam. 2010;2(Ed Supl ):654-6.

http://dx.doi.org/10.9789/2175-5361.2010.v0i0.\%25p

16. Garbin CAS, Lima DP, Dossi AP, Arcieri RM, Rovida TAS. Percepção de adolescentes em relação a doenças sexualmente transmissíveis e métodos contraceptivos. DST J Bras Doenças Sex Tansm. 2010;22(2):60-3.

17. Pereira GS, Borges $\mathrm{Cl}$. Conhecimento sobre HIV/AIDS de participantes de um grupo de idosos, em Anápolis-Goiás. Esc Anna Nery. 2010;14(4):720-5. http://dx.doi.org/10.1590/S1414-81452010000400010

18. Garcia S, Souza FM. Vulnerabilidades ao HIV/aids no contexto brasileiro: iniquidades de gênero, raça e geração. Saúde Soc. 2010;19(Supl 2):9-20.

http://dx.doi.org/10.1590/S0104-12902010000600003

19. Brasil. Ministério da Saúde. Secretaria de Vigilância em Saúde. Programa Nacional de DST e Aids. Manual de adesão ao tratamento para pessoas vivendo com HIV e aids. Brasília: Ministério da Saúde; 2008.

20. Souza MHT, Backes DS, Pereira ADA, Ferreira CLL, Medeiros HMF, Marchiori MRCT. Nível de Conhecimento de um grupo de idosos em relação à Síndrome da Imunodeficiência Adquirida. Av Enferm. 2009;27(1):22-9.

21. Santos AFM, Assis M. Vulnerabilidade das idosas ao HIV/AIDS: despertar das políticas públicas e profissionais de saúde no contexto da atenção integral: revisão de literatura. Rev Bras Geriatr Gerontol. 2011:14(1):147-58. http://dx.doi.org/10.1590/S1809-98232011000100015 